\title{
Cost-effectiveness analysis of One Health surveillance strategies for rabies control
}

\section{Kristyna Rysava, Michael Tildesley}

\author{
University of Warwick
}

\section{Objective}

To evaluate public health and economic impact of intersectoral One Health enhanced surveillance strategies of canine rabies in the Philippines to inform vaccine procurement and provision plans.

\section{Introduction}

Rabies control programmes are being implemented across the Philippines, with a number of islands and provinces on track for the elimination of both human and dog rabies [1,2]. In spite of considerable progress in control programmes, costs of post exposure prophylaxis (PEP) remain high with bite incidence rapidly increasing every year. Indiscriminate PEP administration can strain healthcare budgets, and eventually redirect focus from essential mass dog vaccination campaigns. It is an ethical imperative to improve access to PEP for those at risk; however, under the current framework operating in the Philippines, indiscriminate PEP administration results in excessive expenditure on non-case patients and subsequent financial strains and vaccine shortages, whilst vulnerable communities remain undetected. At the same time, incursions represent an obstacle to achieving and maintaining rabies freedom [3,4] and have been shown to pose a threat to elimination goals [5]. The lack of formal surveillance is the primary cause, leading to late detection of disease at which point substantial secondary transmission within the dog population already occurs. There is, therefore, an urgent need to enhance and streamline surveillance to maximize detection potential for rapid outbreak response and to guide policy decisions regarding public health practice. Workable surveillance criteria are needed for more judicious and effectiveuse of PEP to identify high risk exposures and thus prevent unnecessary risk and further transmission when vaccine stocks are limited.

\section{Methods}

Integrated models that capture epidemiological and health dynamics are essential to evaluate cost-effectiveness of control strategies and have the potential to directly inform rabies control programmes. Here we sought to develop an epidemiological model for rabies transmission within the dog population and from dogs to humans, incorporating information on health-seeking behaviour collected through a longitudinal enhanced surveillance study of dog bite-injury patientsongoing atanti-rabies clinics in Albay province, Philippines.Through computational simulations, we investigated changes in rabies dynamics and economic benefits of three potential surveillance scenarios: (1) current practice of indiscriminate PEP administration with no investigation of biteincident histories, (2) quarantine of suspect dogs identified through bite-histories of patients presenting at clinics and (3) quarantine of suspect dogs with detailed triage of patients and follow up outbreak investigations.

\section{Results}

Utilizing data collected at anti-rabies clinics, we found that bite incidence in Albay is high (monthly mean=796, sd=337) with PEP administered unsystematically. All patients presented at clinics received at least 1 dose of PEP, 95\% of patients received 2 doses and $89 \%$ of patients received 3 doses. Only $3 \%$ of patients received the fourth dose, likely owing to the cost patients are charged for the last dose (first three doses are provided free of charge). Additionally, 17\% received a dose of costly RIG. This is consistent with previous reports of generous use of PEP and RIG in the Philippines [6].

We found that in comparison to the current practices (scenario1) the integrated bite-case management strategies - quarantine of suspect dogs (scenario 2) and quarantine of suspect dogs with detailed triage of patients and outbreak investigations (scenario 3) demonstrated a substantial reduction in costs through savings on expensive PEP and RIG despite additional expenditures on surveillance. The total costs for rabies prevention in humans would be reduced by $47 \%$ and $57 \%$ deploying scenarios 2 and 3 respectively. However, an ongoing risk of human deaths persists for as long as rabies continues to circulate in domestic dog populations. We have, therefore, investigated the impact of dog quarantine on rabies dynamics in the context of disease elimination and persistence. Scenarios 2 and 3 resulted in a clear decline in incidence of both dog and human cases. Moreover, under increased detection of infected dogs through field investigations (scenario 3) rabies appears to persist solely through repeat exogenous incursions. 


\section{Conclusions}

Coalescing our understanding of health landscapes with that of transmission dynamics enables us to evaluate the demand of PEP provisioning, how this demand will evolve across the elimination timeframe, and the effectiveness of individual intervention strategies in preventing human deaths.

Joint investigations foster intersectoral relationships and collaborative investments between public health and veterinary services. Triage of patients and investigations of suspect dogs allows for improved PEP recommendations and reduction of the current unnecessary expenditures whilst active field investigations lead to increased and early detection of rabies in dogs and identification of rabies exposed persons who would otherwise not seek care. Quarantine of rabies suspect dogs appears powerful in curtailing transmission, but large-scale vaccination of dogs is necessary for complete interruption of transmission of the virus and sustained elimination of rabies, given the enduring risk of re-introductions from neighbouring populations [7-9]. However, early detection of incursions is critical and can preclude an undesired outbreak. Integrated One Health approaches of rabies surveillance have the potential to substantially increase case detection $[10,11]$ and ultimately generate vital evidence for verifying freedom from disease [12].

\section{References}

[1] Miranda LM et al., 2017, Transbound Emerg Dis.; [2] Barroga TRM et al., 2018, Trop Med and Inf Dis.; [3] Zinsstag J et al., 2017, Science Translational Medicine.; [4] Bourhy H et al., 2016, PLOS Pathogens.; [5] Tohma K et al., 2016, Genetics and Evolution.; [6] Hampson Ket al., 2015, PLOSNeg Trop Dis.; [7] Putra AAG et al., 2013, Emerg Inf Dis.; [8] Bamaiyi PH et al., 2015, J Vet Advanc.; [9] Windiyaningsih C et al., 2004, J Med Assoc Thai.; [10] Rajeev M et al., in print, Vaccine.; [11] Rysava $\mathrm{K}$ et al., in print, Vaccine.; [12] Hampson K et al., 2016, BioRxiv.

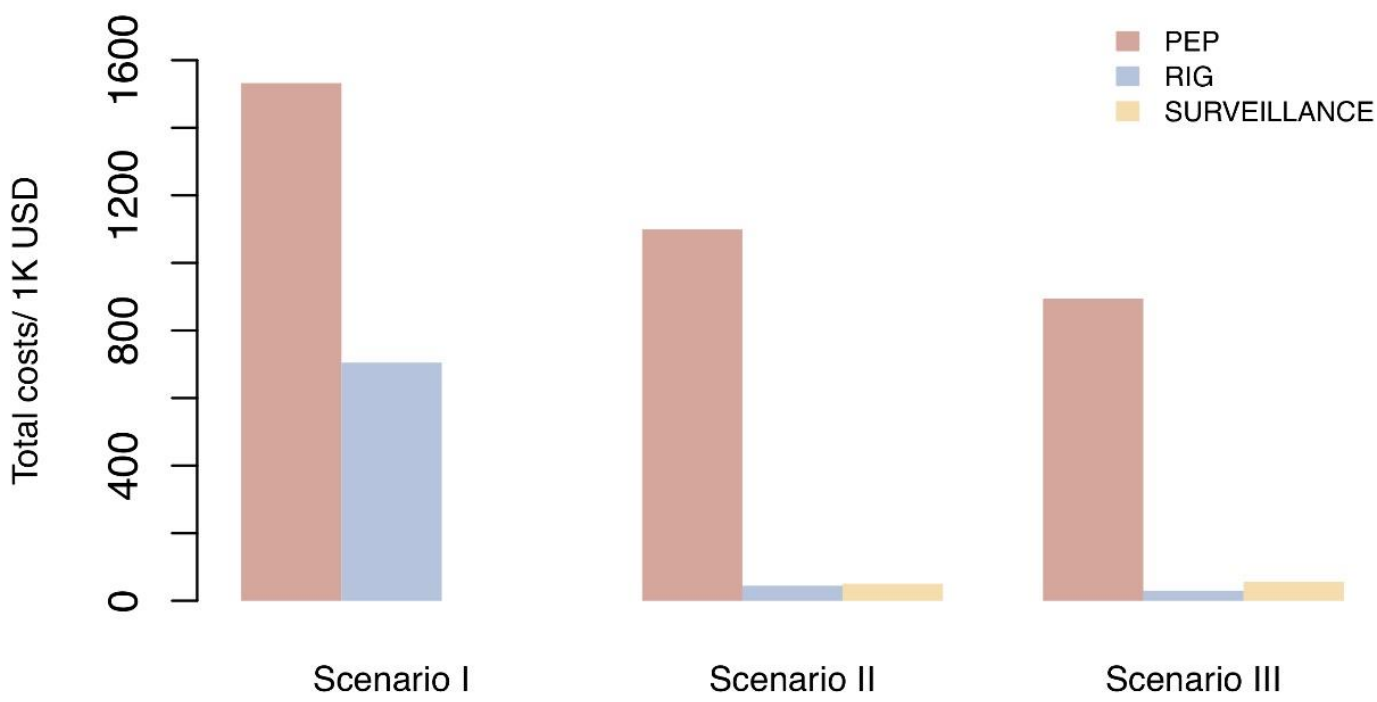

SDS Annual Conference Proceedings 2019. This is an Open Access article distributed under the terms of the Creative Commons AttributionNoncommercial 4.0 Unported License (http://creativecommons.org/licenses/by-nc/3.0/), permitting all non-commercial use, distribution, and reproduction in any medium, provided the original work is properly cited. 